\title{
Construction of a Neutron Monitoring Detector for the COSINE-100 experiment
}

\author{
Govinda Adhikari* \\ on behalf of the COSINE-100 collaboration \\ Department of Physics, Sejong University, Seoul, 05006, Republic of Korea \\ E-mail: adhikari.astro@gmail.com
}

\begin{abstract}
The goal of the COSINE-100 experiment is to confirm or refute the controversial observation of a WIMP Dark Matter like annual modulation by the DAMA/LIBRA experiment. Measurement of neutron flux for dark matter search experiment is important since seasonal modulations of environmental neutrons that interact with the nuclei in the $\mathrm{NaI}(\mathrm{Tl})$ target/detector crystal array might mimic the WIMP-induced signals. To understand environmental neutron flux for the COSINE-100 experiment systematically, we develop a Neutron Monitoring Detector using liquid scintillator and study its energy response, characterize its pulse shape discrimination properties, and measure background from the detector. Here, construction and tests of the Neutron Monitoring Detector for the COSINE-100 experiment are presented.
\end{abstract}

35th International Cosmic Ray Conference - ICRC2017

10-20 July, 2017

Bexco, Busan, Korea

${ }^{*}$ Speaker. 


\section{Introduction}

The DAMA/LIBRA experiment searches for an annual modulation in the detection rate of nuclear recoils in an array of ultra-low-background $\mathrm{NaI}(\mathrm{Tl})$ crystals caused by the Earth's orbital motion through our Galaxy's dark-matter halo. This experiment, which has been operating for over 15 years, has consistently reported a positive signal for an annual modulation with a phase that is consistent with expectations for motion of the earth relative to the galactic rest frame. The statistical significance of the DAMA/LIBRA's annual modulation signal has now reached 9.3 sigma [1,2].

The COSINE collaboration has been developing ultra-low-background $\mathrm{NaI}(\mathrm{Tl})$ crystal detector to search for Weakly Interacting Massive Particle (WIMP). The primary aim of the experiment is to confirm or refute the claimed observation of the DAMA/LIBRA experiment with the same type of crystal. In particular, with the cumulative exposure of the modulation amplitude of the single hit events in the (2-6) keV energy interval measured in $\mathrm{NaI}(\mathrm{Tl})$ target is $(0.0116 \pm 0.0013)$ $\mathrm{cpd} / \mathrm{kg} / \mathrm{keV}$ by DAMA/LIBRA experiment [1]. So, low background environment is the crucial issue for the COSINE-100 experiment. The COSINE-100 experiment is located at the Yangyang Underground Laboratory(Y2L), Korea with the minimum earth overburden of $700 \mathrm{~m}$. There have been continuing debates about whether or not the DAMA annual modulation signal is due to muoninduced neutron signals that are known to be seasonally modulated [8]. So, it is important to understand muon-induced neutrons because neutron-induced nuclear recoils can mimic WIMPnucleon interaction events.

The main source of neutron background are spontaneous fission of ${ }^{238} \mathrm{U}$ in the rocks, $(\alpha, \mathrm{n})$ reactions caused by alpha particles from the decay of ${ }^{238} \mathrm{U}$ and ${ }^{232} \mathrm{Th}$. Additionally, the high energy cosmic ray muons interacting with the shielding materials may generate neutrons inside the shield. Due to possible modulation of environmental neutrons, that can interact with nuclei, neutron monitoring is necessary for the annual modulation study. For this reason, we are constructing Neutron Monitoring Detector (NMD) by using liquid scintillator to install inside the main shielding of the COSINE-100 experiment. Here, we describe an R\&D on construction of NMD for the COSINE-100 experiment.

\section{Experimental Test Bench}

A cylindrical container having $5 \mathrm{~cm}$ length and $4.5 \mathrm{~cm}$ inner diameter and made of $1.5 \mathrm{~cm}$ thick teflon is used for a pulse shape discrimination (PSD) study. Both end of the cell have PMMA window with $10 \mathrm{~mm}$ thickness. Two different types of liquid scintillators are candidates for the PSD study. One is a LAB-based LS and the other is a Di-isopropylnaphthalene $\mathrm{C}_{16} \mathrm{H}_{20}$ based LS which is a commercial product called "Ultima Gold-F (UGF)". The test detector is read out by two 3-inch PMTs (R12669SEL) mounted on each end of the cylinder. The detector is covered with a teflon sheet for better light collection and a dark sheet to prevent light leaking. A $5 \mathrm{~cm}$ thick polyethylene (PE) \& a $5 \mathrm{~cm}$ lead shield the detector to control environmental neutrons and gammas. A picture of the experimental test bench for the PSD study is shown in figure1.

To understand the background level, another detector with 10 times large in volume is installed inside the KIMS-CsI test facility at the Y2L [3]. An available polyethylene shielding maybe good enough to prevent external neutrons. 


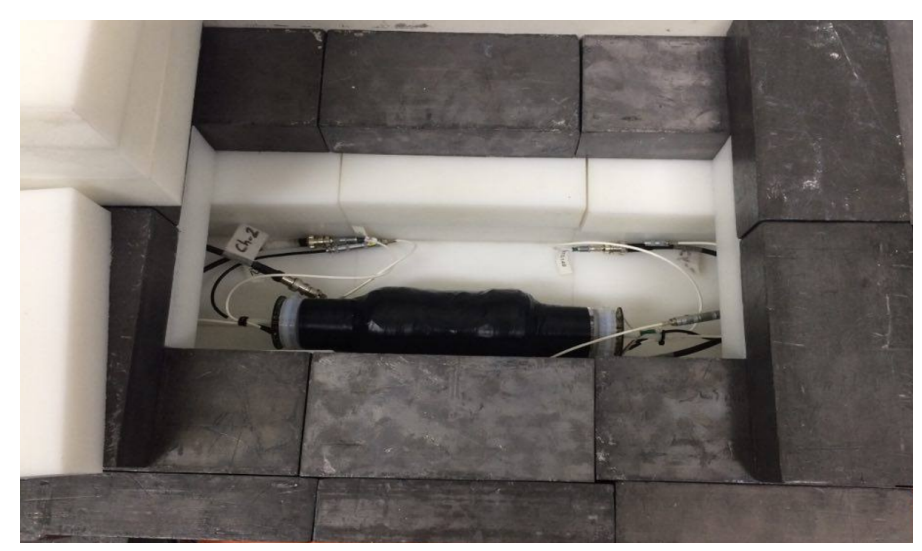

Figure 1: An experimental test bench for pulse shape discrimination study

The $1332 \mathrm{keV}$ gammas from ${ }^{60} \mathrm{Co}$ is used to calibrate the detector. Because of low mass number of the liquid scintillator, it is difficult to see the full peak of the $1332 \mathrm{keV}$ gammas. So, the Compton edge is used for the energy calibration as shown in figure 2 .

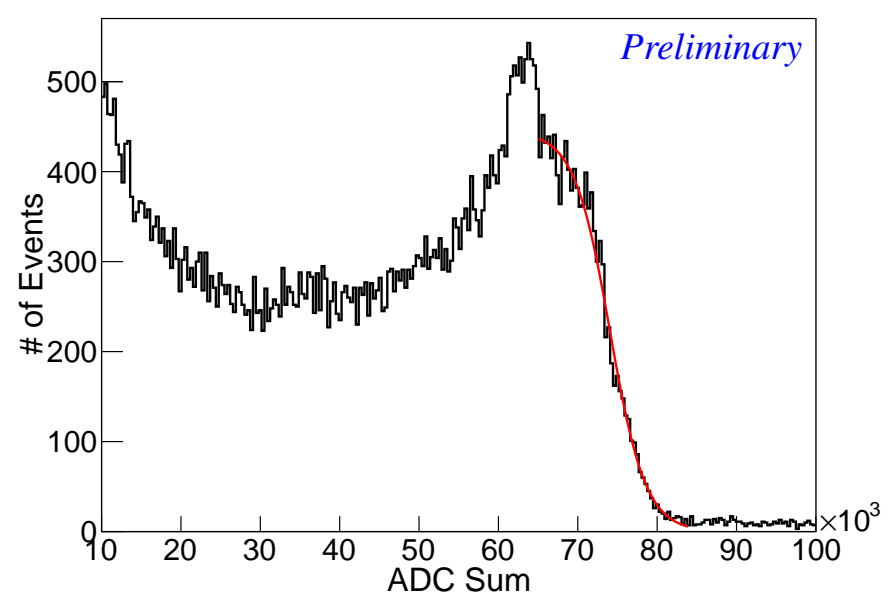

Figure 2: Calibration of the detector using a ${ }^{60} \mathrm{Co}$ external gamma source

\section{Liquid scintillator}

Linear Alkyl Benzene (LAB) is one of the commonly used candidate for the pulse shape discrimination study. The emission spectrum of LAB has the maximum at $340 \mathrm{~nm}$, therefore, it needs to be mixed with a wavelength shifter to adjust wavelength of the emitted optical photons suitable for the photomultiplier tube. We used PPO (2,5-Diphenyloxazole, $\mathrm{C}_{15} \mathrm{H}_{11} \mathrm{NO}$ ) for primary fluor and bis-MSB (1,4-Bis(2-methylstyryl) benzene), $\left(\mathrm{CH}_{3} \mathrm{C}_{6} \mathrm{H}_{4} \mathrm{CH}=\mathrm{CH}_{2}\right)_{2} \mathrm{C}_{6} \mathrm{H}_{4}$ ) for secondary wavelength shifter. The amount of PPO was chosen to be $3 \mathrm{~g} / \mathrm{L}$ and bis-MSB was chosen to be 30 $\mathrm{mg} / \mathrm{L}$ [4]. 
Commercial liquid scintillator cocktail UGF produced by PerkinElmer company is another candidate for pulse shape discrimination R\&D. Di-isopropylnaphthalene (DIN) $\mathrm{C}_{16} \mathrm{H}_{20}$ is the solvent for this cocktail.

\subsection{Pulse Shape Discrimination Technique}

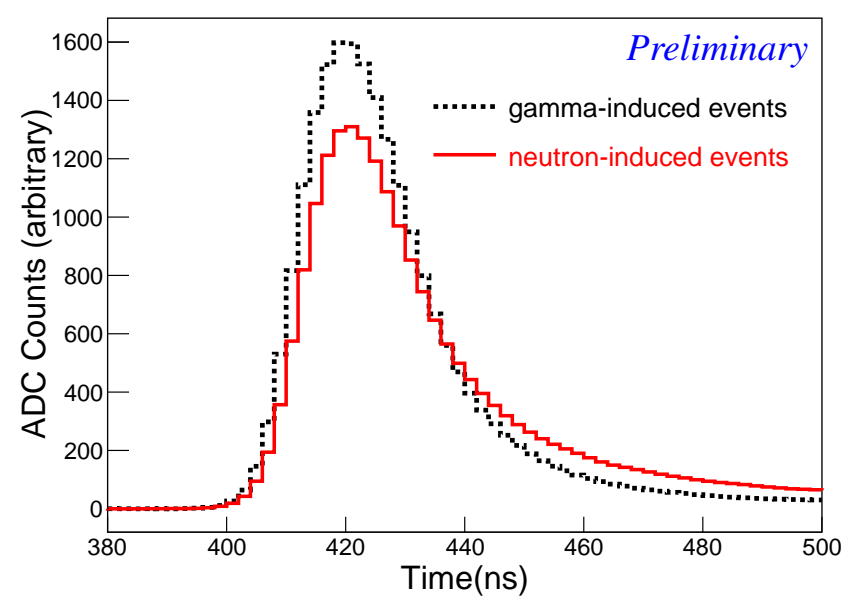

Figure 3: Event shapes of neutrons and gammas in UGF

The PSD method is one of the most important characteristic features of Liquid scintillators . The light emitted from the vast majority of LS consists of two main components; the fast and the slow component. The fast one has a decay constant in the range of a few ns. The slow component has an exponential tail extending to several hundred ns [6]. So, signal shape of fast neutron events is different from gamma events as shown in figure 3. The fast neutron has a longer tail than that of a gamma due to greater de-excitation of different states in the LS [7]. So we can distinguish gamma and neutron event by taking the ratio between the integrated tail charge (Q_tail) to the total charge(Q_total) of the waveform. This ratio parameter is called as a PSD parameter which is widely used for neutron/gamma discrimination.

To compare the PSD performance of the two different LS, we define a figure of merit (FoM) as

$$
F O M=\frac{\Delta m}{\sqrt{s_{1}^{2}+s_{2}^{2}}},
$$

where $\Delta \mathrm{m}$ represents the difference between the fit mean values of the gamma \& neutron distributions, and $s_{1} \& s_{2}$ are their respective standard deviations in the PSD parameter. Therefore, a higher FoM indicates a better discrimination between events induced by gamma \& neutron.

\subsubsection{PSD Performance by different Liquid Scintillator}

PSD powers of LAB-based LS and UGF-based LS are compared by using the technique described in the Section 3.1 using a ${ }^{252} \mathrm{Cf}$ source which emits both gammas and fast neutrons. The PSD parameter (Q_tail/Q_total) is optimized for each sample by integrating the tail charge of pulses 
in each time bin starting from the maximum pulse height bin using the ${ }^{252} \mathrm{Cf}$ source as shown in figure 4. In our measurements, UGF shows the best discrimination power. FoM of the optimized UGF-based LS is measured to be 7.1 at the energy range between $200 \mathrm{keV}$ and $1500 \mathrm{keV}$ while the LAB-based LS shows FoM of 4.0 measured in the same energy range as shown in figure 4.

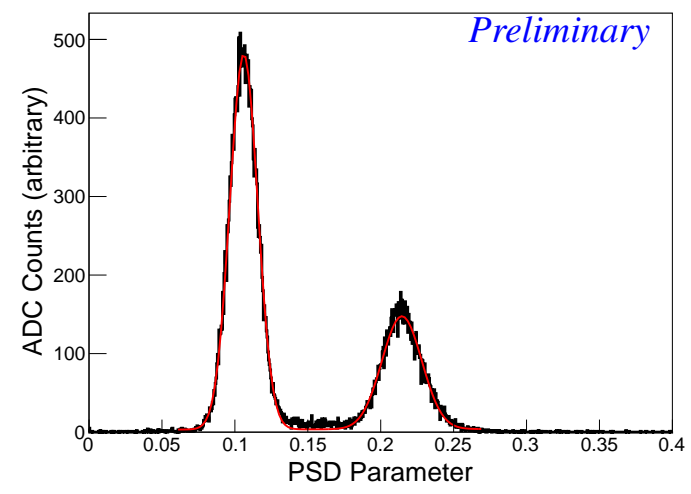

(a)

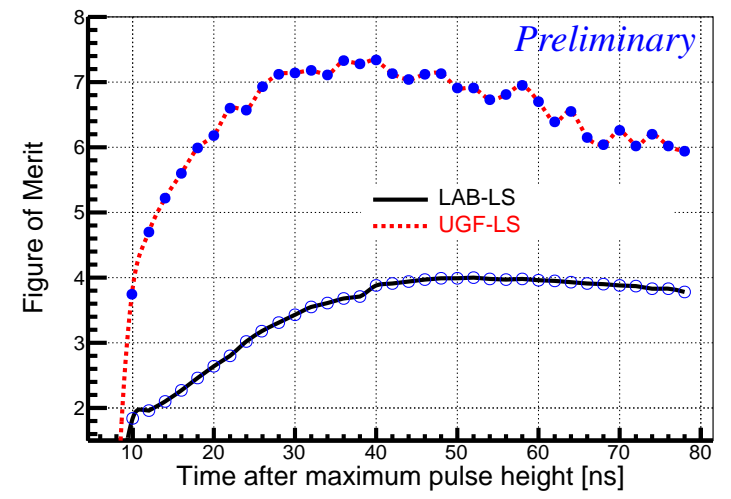

(b)

Figure 4: PSD using a ${ }^{252} \mathrm{Cf}$ source (a) Number of entries as a function of PSD parameter and (b) Optimization of PSD parameter in terms of FoM with different tail charges

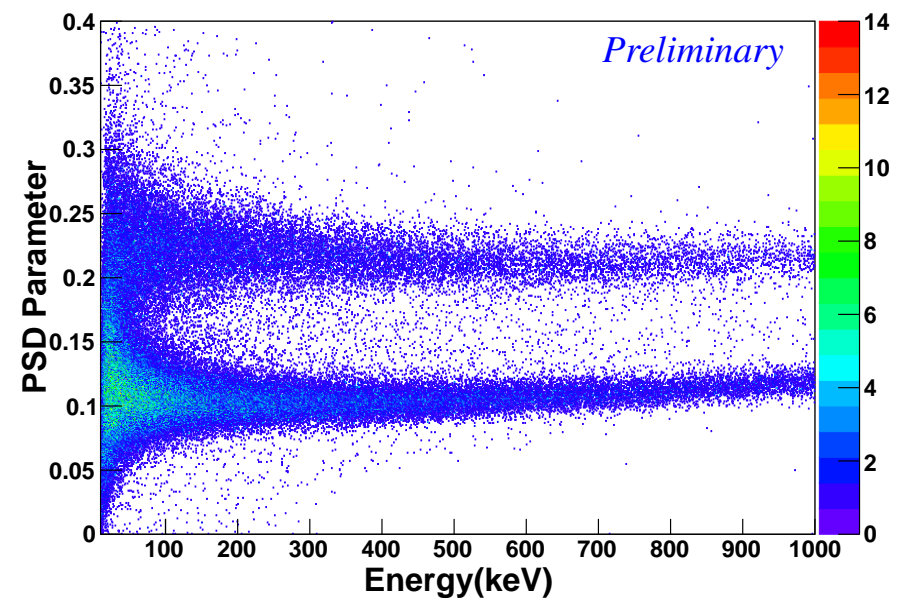

Figure 5: PSD parameter as a function of energy for the UGF detector

Based on the PSD measurements, the UGF LS is the better candidate material for our NMD. The PSD parameter as a function of electron equivalent energy is shown in figure 5. Because of the longer tail of neutron waveform than that of gamma waveform, the band having PSD parameter around $0.22 \& 0.10$ are from neutrons and gammas events respectively.

From this measurement, FoM is 3.2 at $100 \mathrm{keV}$ so that PSD threshold between gamma and neutron band is around $100 \mathrm{keV}$ as shown in figure 5. So, we expect good FoM as well as low PSD threshold for the COSINE-100 NMD which is going to be constructed with a total mass of about 5 $\mathrm{kg}$. 


\section{Natural background}

Natural radioactive decay chains of ${ }^{238} U \&{ }^{232}$ Th are the major background for the LS. The ${ }^{238} \mathrm{U} \&{ }^{232} \mathrm{Th}$ in LS will decay according to each decay chain and will emit alpha or beta. Relative amount of light from heavily ionizing particles like $\alpha$ is larger than that of relativistic beta particles. Therefore, PSD parameter is effective to separate beta and alpha events. The signal from alpha is similar to nuclear recoil signal from neutrons in the NMD. Therefore understanding alpha background for NMD is important. To understand background, detector filled with UGF having mass $0.66 \mathrm{~kg}$ is installed inside the KIMS-CsI shielding test facility at the Y2L [3]. The alpha energy distribution from the detector is as shown in figure 6.

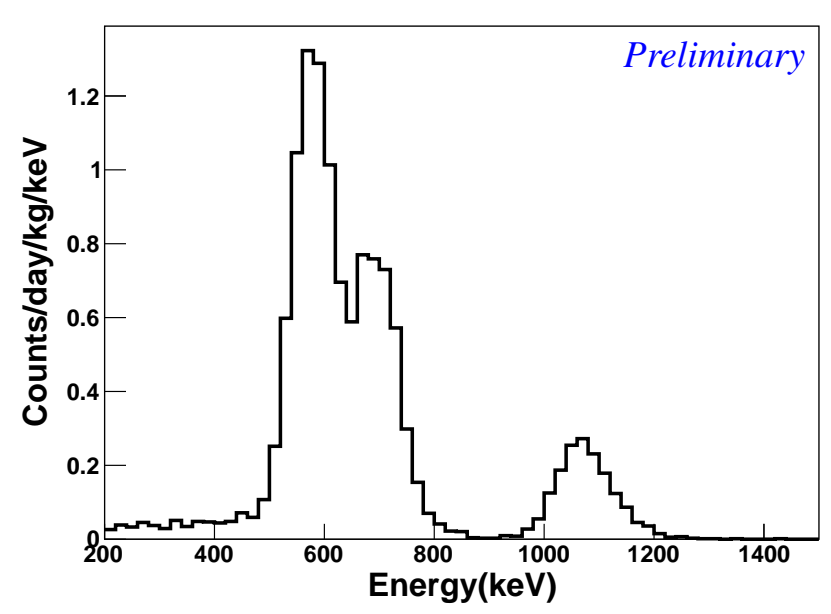

Figure 6: Alpha energy distribution from the UGF detector

It is found that the total alpha background activity is $3.17 \pm 0.4 \mathrm{mBq} / \mathrm{kg}$ which may give a high uncertainty while measuring the neutron flux. Alpha particles from ${ }^{238} \mathrm{U} \&{ }^{232} \mathrm{Th}$ can be estimated by the $\beta-\alpha$ and $\alpha-\alpha$ auto-correlation study. ${ }^{238} \mathrm{U}$ can be evaluated by exploiting the $237 \mu$ s mean lifetime of a ${ }^{214} \mathrm{Po} \alpha$-decay, which immediately follows its production via $\beta$-decay of ${ }^{214} \mathrm{Bi}$. For ${ }^{232} \mathrm{Th}$, time difference between two sequential alpha events from ${ }^{216} \mathrm{Po} \alpha$-decay component comes with a mean time of $209 \mathrm{~ms}$. We found that UGF contains a ${ }^{214}$ Po decay components, corresponding to a $0.4 \pm 0.06 \mathrm{mBq} / \mathrm{kg}$ of ${ }^{238} \mathrm{U}$ contamination. But there is no time dependent exponential components which characterize ${ }^{216} \mathrm{Po}$, so we calculate upper limit of activities $0.02 \mathrm{mBq} / \mathrm{kg}$ of ${ }^{232} \mathrm{Th}$. Fraction of those estimated alpha events are relatively smaller in comparison to the total alpha events. The origin of these alpha background is under study.

It is essential to control alpha background from the detector. Different radio-purification methods searches are ongoing to achieve a higher radio-purity target material. We are focusing to remove ${ }^{238} \mathrm{U} \&{ }^{232} \mathrm{Th}$ chain isotopes which may exist into the liquid scintillator either in ionic or organic or metallic state. 


\section{Summary}

Among various organic scintillators, the Ultima-Gold F cocktail liquid has an advantage of having the best pulse shape discrimination between neutrons and gammas. So, the UGF is one of the strong candidate target material for the measurement of fast neutron flux at the Yangyang Underground laboratory. A Neutron Monitoring Detector will be prepared after understanding purification recipe and will be installed into the COSINE-100 experiment room in near future.

\section{Acknowledgements}

We thank the Korea Hydro and Nuclear Power (KHNP) Company for providing the underground laboratory space at Yangyang. We also acknowlege the following supports : the Institute for Basic Science (IBS) under project code IBS-R016-A1, Republic of Korea; the Alfred P. Sloan Foundation Fellowship, NSF Grants No. PHY-1151795 and PHY-1457995, WIPAC, the Wisconsin Alumni Research Foundation, and Yale University, United States; STFC grant ST/N000277/1, United Kingdom; CNPq, Brazil.

\section{References}

[1] R. Bernabei et al., New results from DAMA/LIBRA, Eur. Phys. J. C 67 (2010) 39.

[2] R. Bernabei et al., Final model independent result of DAMA/LIBRA-phase 1, Eur. Phys. J. C 73 (2013) 2648.

[3] H. S. Lee et al., Development of low background CsI(Tl) crystals for WIMP search, Nuclear Instruments and Methods in Physics Research A, 571:644 (2007).

[4] J. S. Park et al., Production and optical properties of Gd-loaded liquid scintillator for the RENO neutrino detector, Nuclear Instruments and Methods in Physics Research A , 707, pp 45-43(2013).

[5] Klein H et al., Scintillation detectors for fast neutrons, F D 2006 Proceedings of Science, PoS(FNDA2006)

[6] Lombardi P et al., Decay time and pulse shape discrimination of liquid scintillators based on novel solvents, Nuclear Instruments and Methods in Physics Research A, 701, pp. 133-144 (2013).

[7] Blum K et al., DAMA vs. the annually modulated muon background, astro-ph.HE 1110.0857 (2011). 